\title{
A demonstration of donor passivation through direct formation of V-As-i complexes in As-doped Ge1-xSnx
}

\section{Khanam, Afrina}

2020-05-21

Khanam , A , Vohra , A, Slotte , J Makkonen , I, Loo, R, Pourtois , G \& Vandervorst , W 2020 , ' A demonstration of donor passivation through direct formation of $\mathrm{V}$-As-i complexes in As-doped Ge1-xSnx ' , Journal of Applied Physics , vol. 127 , no. 19 , 195703 . https://doi.org/10.1063/5.0003999

http://hdl.handle.net/10138/316043

https://doi.org/10.1063/5.0003999

unspecified

publishedVersion

Downloaded from Helda, University of Helsinki institutional repository.

This is an electronic reprint of the original article.

This reprint may differ from the original in pagination and typographic detail.

Please cite the original version. 


\section{A demonstration of donor passivation through direct formation of $\mathrm{V}-\mathrm{As}_{i}$ complexes in As-doped $\mathrm{Ge}_{1-x} \mathrm{Sn}_{x}$}

Cite as: J. Appl. Phys. 127, 195703 (2020); https://doi.org/10.1063/5.0003999

Submitted: 07 February 2020. Accepted: 30 April 2020 . Published Online: 15 May 2020

Afrina Khanam (D), Anurag Vohra (D), Jonatan Slotte (D), Ilja Makkonen (D), Roger Loo (D), Geoffrey Pourtois, and Wilfried Vandervorst (iD)
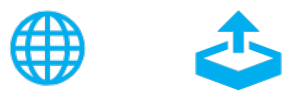

\section{ARTICLES YOU MAY BE INTERESTED IN}

Surface recombination velocities for $4 \mathrm{H}-\mathrm{SiC}$ : Temperature dependence and difference in conductivity type at several crystal faces

Journal of Applied Physics 127, 195702 (2020); https://doi.org/10.1063/5.0007900

Metalorganic chemical vapor phase deposition of AIScN/GaN heterostructures

Journal of Applied Physics 127, 195704 (2020); https://doi.org/10.1063/5.0003095

Influence of substrate misorientation on the optical properties of $\mathrm{Mg}$-doped GaN Journal of Applied Physics 127, 195701 (2020); https://doi.org/10.1063/5.0001482

\section{Lock-in Amplifiers up to $600 \mathrm{MHz}$}
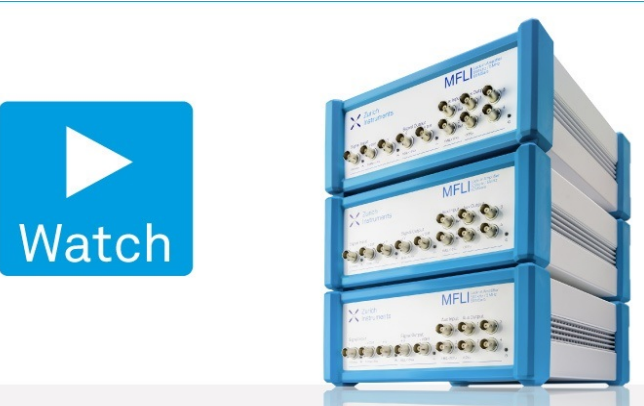


\title{
A demonstration of donor passivation through direct formation of $\mathrm{V}-\mathrm{As}_{i}$ complexes in As-doped $\mathrm{Ge}_{1-x} \mathrm{Sn}_{x}$
}

Cite as: J. Appl. Phys. 127, 195703 (2020); doi: 10.1063/5.0003999

Submitted: 7 February 2020 - Accepted: 30 April 2020 .

Published Online: 15 May 2020

Afrina Khanam, ${ }^{1,2, a)}$ (D) Anurag Vohra, ${ }^{3,4}$ (D) Jonatan Slotte, ${ }^{1,2}$ (iD Ilja Makkonen, ${ }^{2,5}$ (iD Roger Loo, ${ }^{4}$ (D) Geoffrey Pourtois, ${ }^{4,6}$ and Wilfried Vandervorst ${ }^{3,4}$ (D)

\author{
AFFILIATIONS \\ ${ }^{1}$ Department of Applied Physics, Aalto University, P.O. Box 15100, Fl-00076 Aalto, Finland \\ ${ }^{2}$ Department of Physics, University of Helsinki, P.O. Box 43, FI-00014 Helsinki, Finland \\ ${ }^{3}$ Department of Physics and Astronomy, K.U. Leuven, Celestijenlaan 200D, 3001 Leuven, Belgium \\ ${ }^{4}$ Imec vzW, Kapeldreef 75, 3001 Leuven, Belgium \\ ${ }^{5}$ Helsinki Institute of Physics, University of Helsinki, P.O. Box 43, Fl-00014 Helsinki, Finland \\ ${ }^{6}$ Department of Chemistry, Plasmant Research Group, University of Antwerp, B-2610 Wilrijk-Antwerp, Belgium
}

\begin{abstract}
${ }^{a)}$ Author to whom correspondence should be addressed: afrina.khanam@aalto.fi
ABSTRACT

Positron annihilation spectroscopy in the Doppler and coincidence Doppler mode was applied on $\mathrm{Ge}_{1-x} \mathrm{Sn}_{x}$ epitaxial layers, grown by chemical vapor deposition with different total As concentrations $\left(\sim 10^{19}-10^{21} \mathrm{~cm}^{-3}\right)$, high active As concentrations $\left(\sim 10^{19} \mathrm{~cm}{ }^{-3}\right)$, and similar Sn concentrations (5.9\%-6.4\%). Positron traps are identified as mono-vacancy complexes. Vacancy-As complexes, V-As ${ }_{i}$, formed during the growth were studied to deepen the understanding of the electrical passivation of the $\mathrm{Ge}_{1-x} \mathrm{Sn}_{x}$ :As epilayers. Larger monovacancy complexes, $\mathrm{V}-\mathrm{As}_{i}(i \geq 2)$, are formed as the As doping increases. The total As concentration shows a significant impact on the saturation of the number of As atoms $(i=4)$ around the vacancies in the sample epilayers. The presence of $\mathrm{V}$-As $s_{i}$ complexes decreases the dopant activation in the $\mathrm{Ge}_{1-x} \mathrm{Sn}_{x}$ :As epilayers. Furthermore, the presence of $\mathrm{Sn}$ failed to hinder the formation of larger $\mathrm{V}$-As $i_{i}$ complexes and thus failed to reduce the donor-deactivation.
\end{abstract}

Published under license by AIP Publishing. https://doi.org/10.1063/5.0003999

\section{INTRODUCTION}

$\mathrm{Si}$ achieved priority over Ge in device fabrication owing to its larger bandgap, a low surface-state density, and a stable oxide. ${ }^{1}$ However, transistors built out of pure Ge can attain three times higher electron and four times higher hole mobilities due to the low effective masses of the charge carriers compared to those of $\mathrm{Si}$ at low electric fields. ${ }^{2}$ This has led to revive the implementation of Ge in the miniaturization of field effect transistors (FETs), building mid-infrared photodetectors, and designing both logic and photonic device applications. ${ }^{3-6}$ Transistor scaling requires an increase in the concentration of electrically active donor and acceptor atoms to maintain a constant total charge in the source and drain region. ${ }^{7}$ $\mathrm{Ge}_{1-x} \mathrm{Sn}_{x}$ epilayers grown on $\mathrm{Si}$ substrates are playing an interesting role in logic applications for strain engineering and in photonics due to its optical gain. ${ }^{5,8} \mathrm{Ge}_{1-x} \mathrm{Sn}_{x}$ channel pMOSFETs have been shown to achieve higher hole mobility than Ge channel pMOSFETs. ' Epitaxial $\mathrm{Ge}_{1-x} \mathrm{Sn}_{x}$ films mixed with $\mathrm{Si}$ are suitable for the source-drain (S/D) stressors of the n-FETs to maintain sufficient conductivity of the channel between the source and the drain. Milazzo et al. suggested that Excimer Laser Annealing (ELA) can be used to activate implanted Arsenic (As) in Ge to form n-type Ultra-shallow junctions (USJs) with a high active doping concentration of $\sim 10^{20} \mathrm{~cm}^{-3}$. $^{10}$

Point defects and defect complexes in semiconductors can introduce energy levels in the forbidden bandgap that act as compensating centers and charge carrier traps. The intricate electrical and structural properties of defects and defect complexes in Ge have been analyzed by means of Deep Level Transient Spectroscopy (DLTS). ${ }^{11}$ 
Either P-, As-, Sb-, or Bi-doped n-type oxygen lean Ge crystals with doping concentrations of $10^{13}-10^{15} \mathrm{~cm}^{-3}$ were studied by Markevich et al. by means of the capacitance-voltage, the current-voltage techniques, and DLTS. ${ }^{12}$ They showed that E-centers have three charge states in the bandgap: double negative, single negative, and neutral. Nylandsted Larsen and Mesli reported on a third transition level in Sb-doped irradiated Ge, where the $(+/ 0)$ - and $(0 /-)$-states are located below the midgap, and the $(-/--)$-state is in the upper half of the bandgap at $\mathrm{E}_{c}-0.30 \mathrm{eV} .^{11} \mathrm{~A}$ detailed DLTS investigation of radiation damage introduced in p-type Ge by high energy electrons, protons, and alpha particles had been carried out by Petersen et al. to study divacancy defects. They identified one acceptor level associated with the divacancy at $\mathrm{E}_{v}+0.19 \mathrm{eV}$, which likely accounted for both a single and a double acceptor level due to an inverted ordering of the levels. ${ }^{13}$ In a study of intrinsic and extrinsic diffusion of n-type dopants in Ge, Brotzmann and Bracht showed that the simultaneous diffusion of n-type dopants and self-atoms is caused by the presence of doubly negatively charged vacancies $\left(\mathrm{V}^{2-}\right) .{ }^{14}$ They also suggested that the diffusion of all n-type dopants, e.g., $\mathrm{P}, \mathrm{As}$, and Sb, exceeds Ge self diffusion, which reveals an attractive interaction between the dopants and the vacancies, leading to the formation of dopant-vacancy pairs. ${ }^{14}$ They concluded that the activation enthalpy of dopant diffusion for intrinsic conditions decreases with increasing size of the n-type dopants. This indicates an increasing binding energy between the dopant and the vacancy. ${ }^{14}$ Brotzmann et al. reported on the stabilization of donor-vacancy complexes in Ge and the dopant deactivation via the formation of dopant-vacancy complexes in a research on diffusion of self-atoms and n-type dopants in Ge studied by means of controlled multilayer structures. ${ }^{15}$

The Positron Annihilation Spectroscopy (PAS) technique is well suited to analyze open volume point defects in solids. ${ }^{16,17}$ Annealing behavior in electron irradiated Ge was analyzed with PAS by Polity and Rudolf. ${ }^{18}$ The identification of mono- and divacancies with PAS in Ge presents an example for narrow bandgap semiconductors. ${ }^{19,20}$ The existence of the Ge monovacancy at the temperature of $100 \mathrm{~K}$, along with the observation of mobile mono-vacancies pairing up to form divacancies at $200 \mathrm{~K}$, was reported by Slotte et al. ${ }^{19}$ Kuitunen et al. observed clustering of divacancies at elevated temperatures in neutron-irradiated n-type Ge. ${ }^{20}$ Additionally, Slotte et al. showed a free volume of the divacancy size in as-implanted Ge applying PAS. ${ }^{21}$ Recently, Kujala et $a .^{22}$ and Kalliovaara et $a .^{23}$ applied the PAS technique to study vacancies and vacancy clusters in P-, As-, and Sb-doped Ge and in highly As-doped Ge, respectively. Using Czochralski-grown bulk $\mathrm{Ge}$ in which diffusion anneals with As were performed at $680^{\circ} \mathrm{C}$, Kujala et al. showed that the dopant complex accounting for positron trapping is a vacancy multi-donor defect complex with three As atoms around the vacancy. ${ }^{22}$ In the study of highly As-doped $\mathrm{Ge}$, where samples were implanted with $40 \mathrm{keV} \mathrm{As}^{+}$ions, subjected to ELA and recrystallized, a high concentration of vacancy-As complexes larger in open volume than a mono-vacancy was observed by Kalliovaara et l. $^{23}$ Their studies revealed that divacancy complexes contribute substantially to the large inactive fraction of the donors in As-implanted laser annealed Ge. In a previous study, we showed that mono-vacancy size defects are surrounded by one to at least three $\mathrm{P}$ atoms depending on the P-doping level in epitaxial Ge applying PAS. ${ }^{24}$ In a separate study, we focused on the defect analysis on $\mathrm{Ge}_{1-x} \mathrm{Sn}_{x}$ epilayers doped with $\mathrm{P}$ utilizing PAS. ${ }^{25}$ The study revealed that the strong interaction of vacancies with the $\mathrm{P}$ atoms is the reason behind the dominant dopant deactivation in $\mathrm{Ge}_{1-x} \mathrm{Sn}_{x}: \mathrm{P}^{25}$

In this work, we applied PAS to As-doped $\mathrm{Ge}_{1-x} \mathrm{Sn}_{x}$ epilayers to analyze the vacancy-donor complexes. Since compensation of dopants in $\mathrm{Ge}_{1-x} \mathrm{Sn}_{x}$ alloys, highly doped with As, is still unexplored, further investigation is required in order to deepen the understanding of the defect-donor complexes in As-doped $\mathrm{Ge}_{1-x} \mathrm{Sn}_{x}$. This analysis reveals that the defect complexes responsible for positron trapping are mono-vacancies. The extensive formation of $\mathrm{V}-\mathrm{As}_{i}$ complexes appeared with the increase in the As concentration. We also show that the total As concentration has a significant impact on the formation of the vacancy complexes, and the presence of Sn failed to hinder the dopant deactivation.

\section{EXPERIMENTAL DETAILS}

In this study, we implemented the PAS technique to investigate As-doped $\mathrm{Ge}_{1-x} \mathrm{Sn}_{x}$ epilayers. A set of six samples was measured. The thicknesses of the $\mathrm{Ge}_{1-x} \mathrm{Sn}_{x}$ epilayers were $84-88 \mathrm{~nm}$. The epilayers were grown at $315^{\circ} \mathrm{C}$ on top of a $600 \mathrm{~nm}$ Ge template [referred as Ge-virtual substrates (Ge-VS)] on Si (001) substrates using $\mathrm{GeH}_{4}, \mathrm{SnCl}_{4}$, and $\mathrm{AsH}_{3}$ as gas precursors in an industrial standard $300 \mathrm{~mm}$ RP-CVD tool (Intrepid XPTM). The Ge-VSs were made on regular $300 \mathrm{~mm}$ Si wafers using a single step Ge growth, followed by a post-epi anneal at $850^{\circ} \mathrm{C}^{26,27}$ The samples had threading dislocation densities (TDDs) of $\sim 3-6 \times 10^{7} \mathrm{~cm}^{-2}$ as extracted from defect etching and confirmed by electron channeling contrast imaging (ECCI). ${ }^{28,29}$ The growth conditions were kept identical except the partial pressure of $\mathrm{AsH}_{3}$, which was tuned to reach different As-doping levels in $\mathrm{Ge}_{1-x} \mathrm{Sn}_{x}$ layers. The associated material properties of the samples considered in this study are listed in Table I. The total $\mathrm{Sn}$ percentage in as-grown $\mathrm{Ge}_{1-x} \mathrm{Sn}_{x}$ layers was measured using Rutherford backscattering spectrometry (RBS), and the active carrier concentrations were extracted using Micro-Hall effect (MHE) measurements. A Hall scattering factor (HSF) of 1 has been assumed in this study. The total As concentrations, measured by means of Secondary Ion Mass Spectrometry (SIMS), are shown in Fig. 1. Arsenic quantification was done using Ge standards as GeSn standards for SIMS are not available at the moment. The absolute As level in $\mathrm{Ge}_{1-x} \mathrm{Sn}_{x}$ can be slightly off due to the difference in sputter- and ion-yield in $\mathrm{Ge}_{1-x} \mathrm{Sn}_{x}$ layers

TABLE I. Properties of the studied set of samples. At a mass flow ratio of $1.2 \times 10^{-3}$, the growth ceases, and no As is incorporated.

\begin{tabular}{lccc}
\hline \hline \multicolumn{5}{c}{$\begin{array}{c}\text { Mass flow ratio } \\
(\mathrm{Sn})(\%)\end{array}$} & $\left.\begin{array}{c}\text { AsH } \\
3\end{array} \mathrm{GeH}_{4}\right)$ & Active $(\mathrm{As})_{a c}\left(\mathrm{~cm}^{-3}\right)$ & Total $(\mathrm{As})\left(\mathrm{cm}^{-3}\right)$ \\
\hline 6.0 & $5.0 \times 10^{-5}$ & $3.1( \pm 0.4) \times 10^{19}$ & $8.3 \times 10^{19}$ \\
5.9 & $1.0 \times 10^{-4}$ & $5.7( \pm 0.6) \times 10^{19}$ & $1.6 \times 10^{20}$ \\
5.9 & $3.0 \times 10^{-4}$ & $8.6( \pm 0.9) \times 10^{19}$ & $2.5 \times 10^{20}$ \\
6.2 & $1.5 \times 10^{-4}$ & $7.5( \pm 0.9) \times 10^{19}$ & $2.6 \times 10^{20}$ \\
6.4 & $6.0 \times 10^{-4}$ & $5.4( \pm 0.6) \times 10^{19}$ & $4.4 \times 10^{20}$ \\
5.9 & $4.5 \times 10^{-4}$ & $5.9( \pm 0.7) \times 10^{19}$ & $7.4 \times 10^{20}$ \\
\hline \hline
\end{tabular}




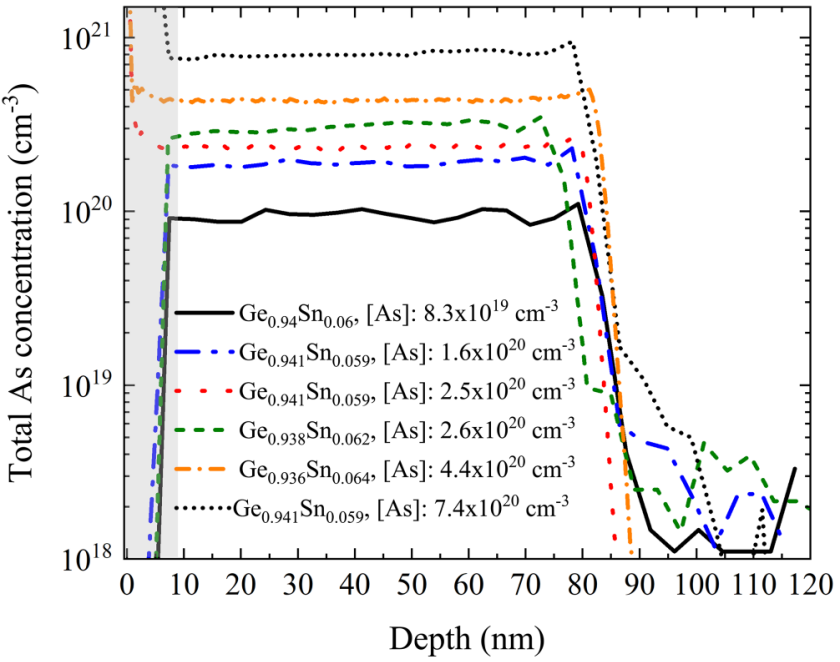

FIG. 1. Total As concentration obtained by SIMS for the studied set of samples. The concentration values in the shaded region cannot be trusted, since the sputtering yield of the SIMS measurement is not stable close to the sample surface.

compared to Ge. However, all samples have similar Sn\%, and a comparison of As levels is, therefore, still meaningful.

In the PAS technique, we use ${ }^{22} \mathrm{Na}$, which emits positrons. Fast positrons are slowed down and electrostatically accelerated to form a variable energy beam. Once the positrons penetrate the sample, they rapidly lose their energy, then live up to a few hundred picoseconds in thermal equilibrium with the lattice and eventually annihilate with an electron. For a monoenergetic positron beam, the average implantation depth is $\sim 10 \mathrm{~nm}$ to $\sim 3 \mu \mathrm{m}$, depending on positron energy. Annihilation of positrons with electrons, either in a delocalized state in the lattice or trapped in a defect, results in the emission of two $\gamma$-quanta with energies of $511 \mathrm{keV}$. In this process, the energy and momentum are conserved. Due to the momentum conservation, the total momentum of the annihilated electronpositron pair is transferred to the annihilation $\gamma$-quanta, leading to a Doppler broadening of the annihilation line. The shape of the $511 \mathrm{keV}$ annihilation line is related to the longitudinal momentum distribution of the annihilating electron-positron pair. The open volume defects can be investigated from the momentum distribution obtained from the annihilation line. A detailed explanation of the PAS technique is discussed in Refs. 16 and 17.

Experiments with Doppler broadening spectroscopy (DOBS), referred to as normal-Doppler, were conducted using a high purity Ge detector (HPGe) with a resolution of $1.3 \mathrm{keV}$ at $511 \mathrm{keV}$. We collected $\sim 10^{6}$ annihilation events per spectrum in a normalDoppler experiment. The energies of the mono-energetic positrons were varied from 0.5 to $25 \mathrm{keV}$. Two shape parameters, conventionally designated as the $S$ - and $W$ - parameters, were used to characterize the spectra. The low momentum parameter $S$, which is also called the valence annihilation parameter, is the fraction of counts in the central region of the annihilation line. On the other hand, the high-momentum parameter $W$, also known as the core annihilation parameter, is the fraction of the counts in the wing region of the annihilation line. ${ }^{16}$

We set the $S$ integration energy window as $|p|<0.46$ a.u., whereas the $W$ integration window was 1.6 a.u. $<|p|<3.9$ a.u. Here, $p$ denotes the longitudinal Doppler shift in terms of momentum and a.u. signifies the atomic units. An increase (decrease) in the $S(W)$ parameter compared to a defect free reference usually indicates the existence of vacancy defects. The measured annihilation parameters $S$ and $W$ are superpositions of the different annihilation states in the lattice, ${ }^{16}$

$$
\begin{gathered}
S=\eta_{B} S_{B}+\sum_{i} \eta_{D i} S_{D i}, \\
W=\eta_{B} W_{B}+\sum_{i} \eta_{D i} W_{D i} .
\end{gathered}
$$

Here, $\eta_{B}$ is the fraction of positrons annihilating in the bulk state, and $\eta_{D i}$ is the fraction of positrons annihilating in the defect state $i$. $S_{B}\left(W_{B}\right)$ and $S_{D i}\left(W_{D i}\right)$ are the bulk and defect parameters, respectively. If a sample contains only two annihilation states, the $W(S)$-plot of the measurement forms a segment of a line between these states, e.g., bulk and defect states. A deviation from the line between the defect and bulk state indicates that positrons annihilate in more than two annihilation states.

2D-coincidence Doppler broadening spectroscopy (CDOBS), referred to as coincidence-Doppler, is an efficient technique to identify vacancy defects and the chemical surroundings of vacancies in the case of vacancy-complexes. After completion of normalDoppler experiments, coincidence-Doppler measurements were done in order to deepen the understanding of the structure of the vacancy defects. Two HPGe detectors were adopted to detect the Doppler broadening of the $511 \mathrm{KeV}$ annihilation line, in order to measure the momentum intensity of the annihilating electronpositron pairs. The trapping of positrons to vacancies leads to narrowing of the annihilation peak. The more vacancies in the lattice, the lower the contribution from core electrons. This results in a reduction of intensities in the high-momentum region of the annihilation line, due to the missing contribution from the absent core electrons. The resolution of the HPGe detector system in the coincidence setup was $1.0 \mathrm{keV}$ at $511 \mathrm{keV}$. A positron implantation energy of $4.5 \mathrm{keV}$ was used to maximize annihilation in the epilayer for the CDOBS measurements. Approximately, $12 \times 10^{6}$ counts for a single spectrum were collected in this experiment. A p-type positron trap free Ge bulk reference sample was used for normalization of the data.

\section{RESULTS}

Figure 2 shows the $W$ parameters as a function of positron implantation energy for a representative set of samples. The average positron implantation depth calculated for Ge is shown on the top axis. At the positron implantation energies of $3.5-6 \mathrm{keV}$, the contribution to the annihilation comes predominantly from the epilayer. Shorter $\mathrm{e}^{+}$diffusion lengths yield steeper slopes in the energy interval of $0-4 \mathrm{keV}$. The samples with a higher total As concentration 


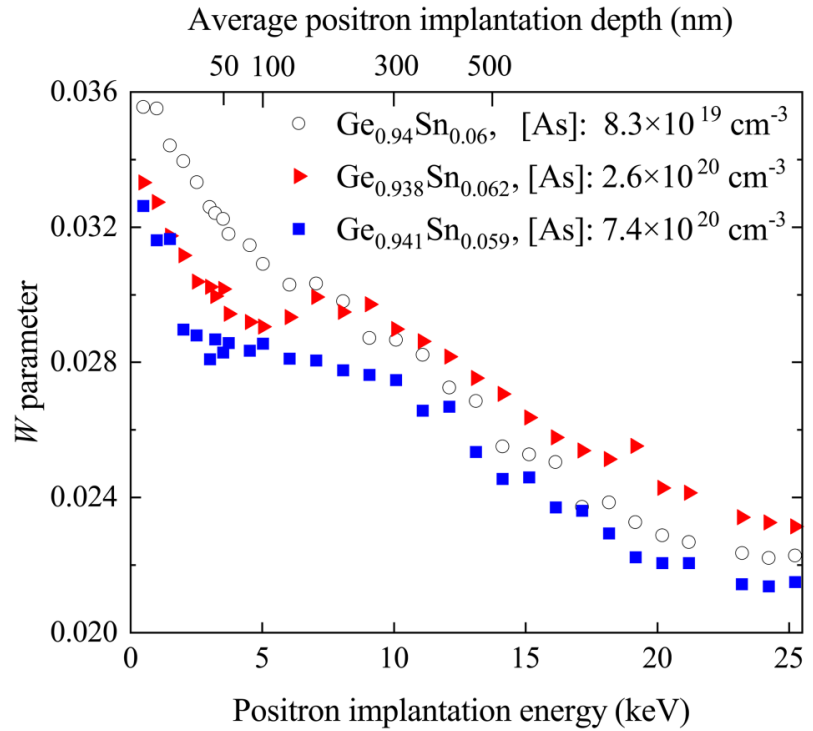

FIG. 2. $W(E)$ plots for samples with different total As concentrations. The error in the $W$ parameter is approximately \pm 0.0001 . The top axis presents the average positron implantation depth calculated for $\mathrm{Ge}$.

contain higher vacancy concentrations, which results in shorter diffusion lengths and a visible plateau in the $W$ parameter in the energy interval of $3.5-6 \mathrm{keV}$, since positrons are getting trapped in the vacancies in the epilayer. The epitaxial layer in the $\mathrm{Ge}_{0.94} \mathrm{Sn}_{0.06}$ sample, with a total As concentration of $8.3 \times 10^{19} \mathrm{~cm}^{-3}$ (marked with open black circles), is challenging to trace out in the energy interval of 3.5-6 keV due to the longer $\mathrm{e}^{+}$diffusion length. This indicates a lower $\mathrm{e}^{+}$trap concentration due to a lower defect concentration in the samples with a lower total As concentration. The $\mathrm{Ge}_{0.94} \mathrm{Sn}_{0.06}$ sample shows a higher $W$ parameter value of $\sim 0.036$, at the energies of $0-2 \mathrm{keV}$ compared to the other two samples shown in Fig. 2. A similar surface behavior was observed for the $\mathrm{Ge}_{0.94} \mathrm{Sn}_{0.059}$ sample grown with a total As concentration of $1.6 \times 10^{20} \mathrm{~cm}^{-3}$ (Table I).

Figure 3 presents the $W$ parameter as a function of the $S$ parameter for the same samples as in Fig. 2. The $\mathrm{e}^{+}$annihilation states at the surface, in the epitaxial layer, in the Ge-VS, and in the substrate are indicated for samples with different total As concentrations. However, no clear state in the epilayer is noticeable for the $\mathrm{Ge}_{0.94} \mathrm{Sn}_{0.06}$ sample epilayer with a total As concentration of $8.3 \times 10^{19} \mathrm{~cm}^{-3}$. The surface state in the $\mathrm{Ge}_{0.94} \mathrm{Sn}_{0.06}$ sample is different from the other two samples in Fig. 3.

Figure 4 shows the ratio of annihilation line intensities scaled to a p-type defect free Ge reference for samples with different total As concentrations obtained from CDOBS measurements. Two ratio curves from prior studies are shown. ${ }^{22,23}$ Kujala et al. found monovacancy complexes $\mathrm{V}$-As $s_{i}$ in bulk Ge. ${ }^{22}$ Kalliovaara et al. observed divacancy complexes $\mathrm{V}_{2}-\mathrm{As}_{i}$ in highly As-doped Ge. ${ }^{23}$ The present $\mathrm{Ge}_{1-x} \mathrm{Sn}_{x}$ :As epilayers resemble the $\mathrm{V}-\mathrm{As}_{i}$ behavior shown by Kujala et al. (Fig. 4). ${ }^{22}$ The corresponding ratio curves, for experiments done by Kujala et al. and for this study, exhibit a peak at

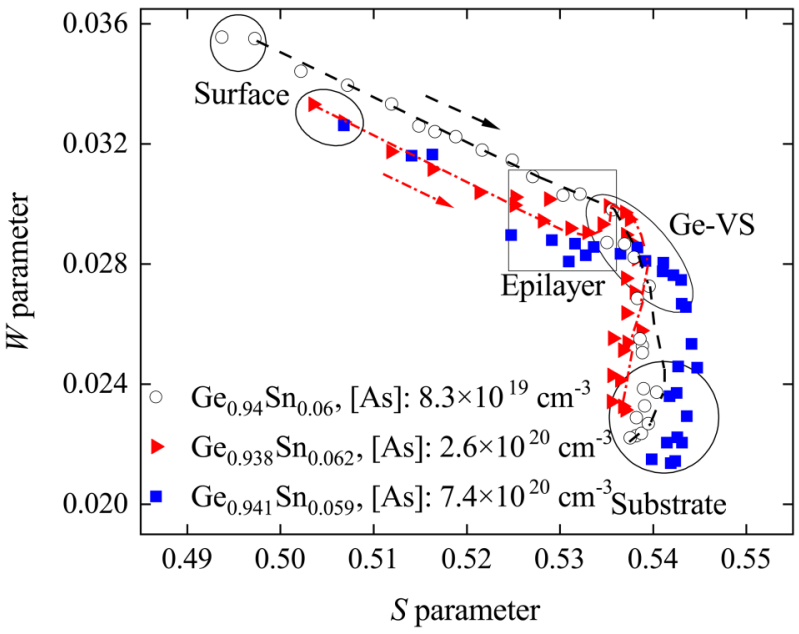

FIG. 3. The $W(S)$ plot for three samples with different total As concentrations. Lines to guide the eyes are drawn for a better understanding of the $\mathrm{e}^{+}$trapping in the epilayers. The arrows indicate the increasing $\mathrm{e}^{+}$implantation energy. The errors for the $S$ and $W$ parameters are approximately \pm 0.0005 and \pm 0.0001 , respectively. Surface, epilayer, Ge-Vs, and substrate states are marked with different shapes.

$\sim 1.2$ a.u. When the positron wave function is localized in a mono-vacancy, the overlap of the positron wave function with the anisotropic electron momentum distribution in the diamond structure is reduced, which causes the peak at $\sim 1.2$ a.u. ${ }^{30}$ With

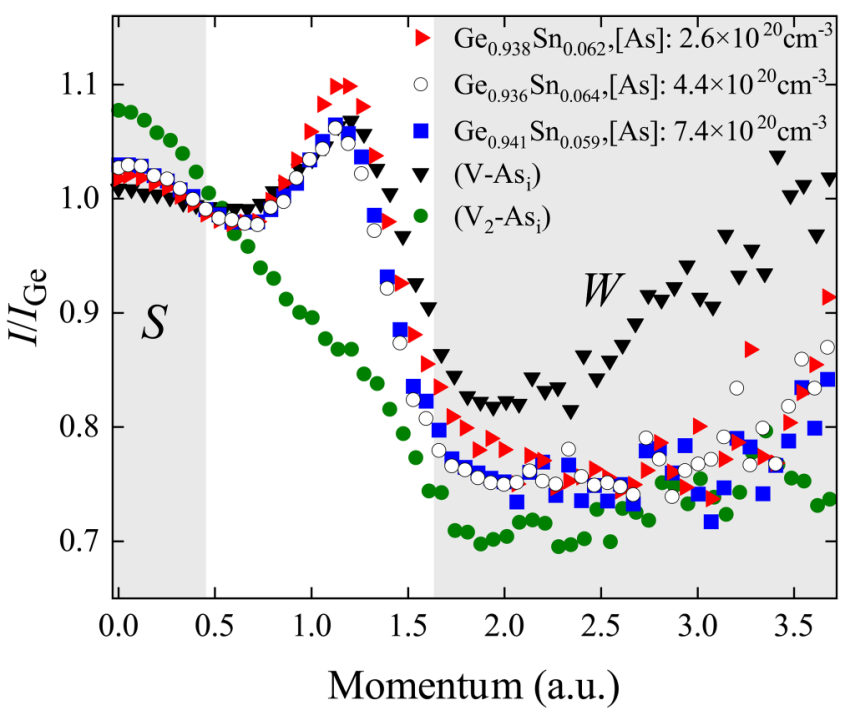

FIG. 4. Intensity ratio for three different samples with CDOBS. The data were normalized to the data from a p-type defect free $\mathrm{Ge}$ sample. Two ratio curves from earlier studies are presented for comparison. $\mathrm{V}-\mathrm{As}_{i}$ was included from Ref. 22, whereas $V_{2}-A s_{i}$ was included from Ref. 23 . The $S$ - and the $W$-parameter windows are indicated with the shaded regions. 
the increasing size of the open volume in the vacancy defects (e.g., $\mathrm{V}_{2}$ ), the contribution from core electrons in the lattice decreases, while the contribution from valence electrons increases. This results in the increase of the $S$ parameter value and hence in the diminishing of the peak at $\sim 1.2$ a.u., as documented by Kalliovaara et al. ${ }^{23}$ From the result presented above, we conclude that the corresponding positron traps in the $\mathrm{Ge}_{1-x} \mathrm{Sn}_{x}$ :As epilayers are mono-vacancy size defects.

Intensities at high momenta, 1.6 a.u. $<|p|<3.9$ a.u., correspond to positrons annihilating mostly with core electrons. The electronic configuration of $\mathrm{Ge}$ is $[A r] 3 d^{10} 4 s^{2} 4 p^{2}$, while the electronic structure of As is $[A r] 3 d^{10} 4 s^{2} 4 p^{3}$. The largest contribution in the high-momentum region comes from the $3 d$ electron shells. A vacancy forms when an atom from the lattice is missing. The decreased intensity in the high-momentum region for the $\mathrm{Ge}_{1-x} \mathrm{Sn}_{x}$ :As samples relates to the reduced overlap of the positron wave function with the $3 d$ electrons. Due to the similar electronic configuration of $\mathrm{Ge}$ and As, the spectra are indistinguishable from each other in the high-momentum region.

\section{DISCUSSION}

$\mathrm{Ge}$, as well as $\mathrm{Si}$, has the diamond lattice structure; i.e., each Ge atom in the lattice has four nearest neighbors. During growth, vacancies can be abundantly formed in the lattice. These vacancies are mobile ${ }^{19}$ until trapped by an impurity atom, in the case of $\mathrm{n}$-type doping by a group-V atom. Brotzmann and Bracht suggested that a doubly negatively charged vacancy forms a defect complex with a positively charged As atom. ${ }^{14}$ They reported on the existence of neutral vacancy-donor complexes $\left(\mathrm{V}-\mathrm{As}_{2}\right)^{0}$ under extrinsic doping since the diffusion of $n$-type dopants is strongly enhanced due to the mobility of the singly negatively charged dopant-vacancy pair $(\mathrm{V}-\mathrm{As})^{-}$. Ranki et al. $^{31}$ observed the formation of immobile $\mathrm{V}-\mathrm{As}_{3}$ complexes from mobile $(\mathrm{V}-\mathrm{As})^{-}$pairs and $\mathrm{V}-\mathrm{As}_{2}$ complexes through the so-called ring mechanism ${ }^{32}$ after electron irradiation of highly As-doped Si. In a similar fashion, Kujala et al. observed $\mathrm{V}-\mathrm{As}_{3}$ complexes in Czochralski-grown bulk Ge after in-diffusion of As at $680^{\circ} \mathrm{C}$. $^{22}$

In epitaxial CVD growth, the $\mathrm{V}-\mathrm{As}_{i}$ complexes can form directly as well as through diffusion. Hence, in addition to $\mathrm{V}$-As, $\mathrm{V}-\mathrm{As}_{2}$, and $\mathrm{V}-\mathrm{As}_{3}, \mathrm{~V}-\mathrm{As}_{4}$ complexes can also be formed. With a dopant concentrations of $10^{19}-10^{21} \mathrm{~cm}^{-3}$ in the $\mathrm{Ge}_{1-x} \mathrm{Sn}_{x}$ :As layers, the estimated vacancy concentration is also of the order of $10^{19}-10^{21} \mathrm{~cm}^{-3}$. Increasing the amount of As in the epitaxial growth process increases the probability of a higher number of As atoms in the mono-vacancy complexes.

$\mathrm{Sn}$ belongs to the group-IV elements in the periodic table and is, therefore, not electrically active as are the group- $\mathrm{V}$ atoms. The $\mathrm{Sn}$ atom is also somewhat larger in size than the As atom. In our present samples, there are both high concentrations of As $\left(10^{19}-10^{21} \mathrm{~cm}^{-3}\right)$ and a high concentration $(\sim 6.0 \%)$ of $\mathrm{Sn}$. With the high Sn concentration in the Ge lattice, the probability of an $\mathrm{Sn}$ atom next to the $\mathrm{V}-\mathrm{As}_{i}$ complex is considerable. In addition, we also documented that in a pure V-Sn complex, the Sn atom relaxes towards the vacancy, creating a split vacancy configuration. ${ }^{25}$ Furthermore, according to calculations, this V-Sn complex does not trap positrons, due to the reduction in the open volume as the
Sn atom relaxes inward. ${ }^{25}$ It should also be noted that although these calculations indicate that the stability of the $\mathrm{V}-\mathrm{Sn}_{j}$ complex increases slightly with an increasing number of $\mathrm{Sn}$ atoms next to the vacancy, the group- $\mathrm{V}$ dopant complexes $\mathrm{V}-\mathrm{D}_{i}$, where $\mathrm{D}=\{\mathrm{P}, \mathrm{As}, \mathrm{Sb}\}$, are energetically favored over $\mathrm{V}-\mathrm{D}_{i}-\mathrm{Sn}_{j}$ and $\mathrm{V}-\mathrm{Sn}_{j}$ in an analogous configuration. However, from a PAS perspective, different $\mathrm{V}-\mathrm{P}_{i}$ complexes are easier to distinguish from each other, since the electronic configuration of $\mathrm{P}$ differs significantly from Ge (outermost core electrons $2 p$ in $\mathrm{P}$ and $3 d$ in $\mathrm{Ge}$ ), and the atom itself is smaller in size than the lattice atoms Ge and Sn. It is evident from the high-momentum region in Fig. 4 that the intensities for the different samples are very similar in the $p>2.0$ a.u. This is expected since the core electronic configuration of As is similar to Ge.

Figure 5 shows the average $W$ parameter and the active As concentration as a function of the total As concentration in the epitaxial $\mathrm{Ge}_{1-x} \mathrm{Sn}_{x}$ layers. A decreasing trend in the $W$ parameter is seen. The $W$ parameter starts to saturate at the total As concentration of $3.5 \times 10^{20} \mathrm{~cm}^{-3}$. With an increasing amount of As in the growth process, the vacancies created in the growth have more As available to be bound to the vacancy. Hence, at a lower total As concentration, the positrons are trapped by a distribution of different $\mathrm{V}-\mathrm{As}_{i}$ complexes, and these complexes could also contain $\mathrm{Sn}$ atoms. However, when the total As concentration is increased, the positrons annihilate in complexes with a higher number of As dopants. When the $W$-parameter saturates, the number of As atoms in the dominating positron trap reaches a maximum; i.e., $\mathrm{V}-\mathrm{As}_{4}$ complexes dominate the positron annihilation. It should be noted that due to the very high dopant concentration, the concentration of vacancies is above the positron saturation trapping limit; i.e., all positrons annihilate in the vacancy complexes. Hence, the PAS technique loses its sensitivity for the defect concentration when the defect concentration is above $\sim 5 \times 10^{18} \mathrm{~cm}^{-3} .{ }^{16}$

As the $W$ parameter starts to saturate in Fig. 5, the concentration of active As dopants starts to decrease. Previous studies have

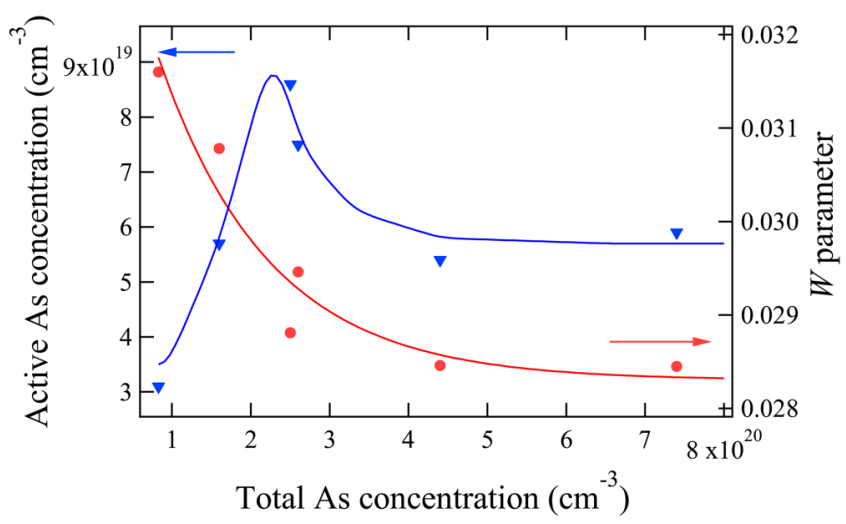

FIG. 5. Active As concentrations and $W$ parameters in the sample epilayers as a function of the total As concentration. The average $W$ parameters have been calculated from the energy interval $3.5-5 \mathrm{keV}$, where the positron localization to the mono-vacancy defects was at a maximum (Fig. 2). The error bars of $W$ parameters are \pm 0.0001 . A line to guide the eyes is used both for the active As concentration and for the $W$ parameter. 
shown ${ }^{23-25}$ that vacancy complexes are the main compensating defects in highly n-type Ge. Hence, we conclude that with both high vacancy concentrations and a large number of As atoms available close to the surface during the growth, the vacancies at a higher total As concentration trap more dopants. Eventually, the $\mathrm{V}-\mathrm{As}_{4}$ complex starts to dominate both the trapping of positrons and the compensation of As dopants. Therefore, the concentration of active As dopants starts to decrease above a total As concentration of approximately $3.5 \times 10^{20} \mathrm{~cm}^{-3}$. The trend in Fig. 5 was not observed in our previous study of $\mathrm{Ge}_{1-x} \mathrm{Sn}_{x}: \mathrm{P}^{25}$ This further strengthens the claim that the binding energy of the vacancy to the dopant increases with an increase in the size of the dopant. ${ }^{14,25}$ Furthermore, the presence of Sn fails to thwart the dopant deactivation, as was observed for P doping in Ref. 25. Reaching carrier concentrations above $10^{20} \mathrm{~cm}^{-3}$ is still a challenge from a production point of view using this growth technique. A possible solution to achieve such high active dopant concentrations could be co-doping of $\mathrm{Ge}_{1-x} \mathrm{Sn}_{x}$ epilayers with, e.g., $\mathrm{P}$ and As, where one of the dopants would be energetically favored to form complexes with the vacancies and the other dopant could contribute to the conductivity.

\section{SUMMARY}

In conclusion, Doppler broadening and coincidence Doppler broadening spectroscopy were applied to examine vacancy defects in As-doped $\mathrm{Ge}_{1-x} \mathrm{Sn}_{x}$ epilayers. The findings from this research suggest that mono-vacancy complexes $\mathrm{V}-\mathrm{As}_{i}$ are responsible for the passivation of dopants in the highly As-doped $\mathrm{Ge}_{1-x} \mathrm{Sn}_{x}$ samples. The total As concentration and hence the number of As atoms at the sample surface during growth show a significant impact on the dominating vacancy defect. Furthermore, the analysis also suggests that the presence of $\mathrm{Sn}$ fails to enhance the donor activation.

\section{DATA AVAILABILITY}

The data that support the findings of this study are available from the corresponding author upon reasonable request.

\section{REFERENCES}

${ }^{1}$ E. Haller, Mater. Sci. Semicond. Process. 9, 408 (2006).

${ }^{2}$ C. Claeys and E. Simoen, Germanium-Based Technologies: From Materials to Devices (Elsevier, London, 2011).

${ }^{\mathbf{3}}$ K. Brunner, Rep. Prog. Phys. 65, 27 (2002).

${ }^{4}$ G. He and H. A. Atwater, Phys. Rev. Lett. 79(10), 1937-1940 (1997).

${ }^{5}$ R. Loo, B. Vincent, F. Gencarelli, C. Merckling, A. Kumar, G. Eneman, L. Witters, W. Vandervost, M. Caymax, M. Heyns, and A. Thean, ECS J. Solid State Sci. Technol. 2(1), N35-N40 (2013).

${ }^{6}$ D. P. Brunco, B. De Jaeger, G. Eneman, J. Mitard, G. Hellings, A. Satta, V. Terzieva, L. Souriau, F. E. Leys, G. Pourtois, M. Houssa, G. Winderickx, E. Vrancken, S. Sioncke, K. Opsomer, G. Nicholas, M. Caymax, A. Stesmans,
J. Van Steenbergen, P. Mertens, M. Meuris, and M. M. Heyns, J. Electrochem. Soc. 155(7), H552-H561 (2008).

${ }^{7}$ R. C. Ropp, Solid State Chemistry (Elsevier, Amsterdam, 2003), p. 340.

${ }^{8}$ R. Soref, J. Kouvetakis, J. Tolle, J. Menendez, and V. D'Costa, J. Mater. Res. 22(12), 3281-3291 (2007).

${ }^{9}$ X. Gong, G. Han, F. Bai, S. Su, P. Guo, Y. Yang, R. Cheng, D. Zhang, G. Zhang, C. Xue, B. Cheng, J. Pan, Z. Zhang, E. S. Tok, D. Antoniadis, and Y. Yeo, IEEE Electron Device Lett. 34(3), 339-341 (2013).

${ }^{10}$ R. Milazzo, E. Napolitani, G. Impellizzeri, G. Fisicaro, S. Boninelli, M. Cuscunà, D. De Salvador, M. Mastromatteo, M. Italia, A. La Magna, G. Fortunato, F. Priolo, V. Privitera, and A. Carnera, J. Appl. Phys. 115, 053501 (2014).

${ }^{11}$ A. Nylandsted Larsen and A. Mesli, Physica B 401, 85 (2007).

${ }^{12}$ V. Markevich, I. Hawkins, A. Peaker, K. Emtsev, V. Emtsev, V. Litvinov, L. Murin, and L. Dobaczewski, Phys. Rev. B 70, 235213 (2004).

${ }^{13}$ M. C. Petersen, A. Nylandsted Larsen, and A. Mesli, Phys. Rev. B 82, 075203 (2010).

${ }^{14}$ S. Brotzmann and H. Bracht, J. Appl. Phys. 103, 033508 (2008).

${ }^{15} \mathrm{~S}$. Brotzmann, H. Bracht, J. Lundsgaard Hansen, A. Nylandsted Larsen, E. Simoen, E. E. Haller, J. S. Christensen, and P. Werner, Phys. Rev. B 77, 235207 (2008).

${ }^{\mathbf{1 6}}$ F. Tuomisto and I. Makkonen, Rev. Mod. Phys. 85, 1583 (2013).

17. Slotte, I. Makkonen, and F. Tuomisto, in Characterisation and Control of Defects in Semiconductors, edited by F. Tuomisto (The Institution of Engineering and Technology, London, 2020), pp. 263-284.

${ }^{18}$ A. Polity and F. Rudolf, Phys. Rev. B 59, 10025 (1999).

19 J. Slotte, S. Kilpeläinen, F. Tuomisto, J. Räisänen, and A. Nylandsted Larsen, Phys. Rev. B 83, 235212 (2011).

${ }^{20}$ K. Kuitunen, F. Tuomisto, J. Slotte, and I. Capan, Phys. Rev. B 78, 033202 (2008).

${ }^{21}$ J. Slotte, M. Rummukainen, F. Tuomisto, V. P. Markevich, A. R. Peaker, C. Jeynes, and R. M. Gwilliam, Phys. Rev. B 78, 085202 (2008).

${ }^{22}$ J. Kujala, T. Südkamp, J. Slotte, I. Makkonen, F. Tuomisto, and H. Bracht, J. Phys. Condens. Matter 28, 335801 (2016).

${ }^{23}$ T. Kalliovaara, J. Slotte, I. Makkonen, J. Kujala, F. Tuomisto, R. Milazzo, G. Impellizzeri, G. Fortunato, and E. Napolitani, Appl. Phys. Lett. 109, 182107 (2016).

${ }^{24}$ A. Vohra, A. Khanam, J. Slotte, I. Makkonen, G. Pourtois, R. Loo, and W. Vandervorst, J. Appl. Phys. 125, 025701 (2019).

${ }^{25}$ A. Vohra, A. Khanam, J. Slotte, I. Makkonen, G. Pourtois, C. Porret, R. Loo, and W. Vandervorst, J. Appl. Phys. 125, 225703 (2019).

${ }^{26}$ V. Terzieva, L. Souriau, M. Caymax, D. Brunco, A. Moussa, S. Van Elshocht, R. Loo, F. Clemente, A. Satta, and M. Meuris, Thin Solid Films 517, 172 (2008).

${ }^{27}$ G. Wang, R. Loo, E. Simoen, L. Souriau, M. Caymax, M. M. Heyns, and B. Blanpain, Appl. Phys. Lett. 94, 102115 (2009).

${ }^{28}$ A. Schulze, L. Strakos, T. Vystavel, R. Loo, A. Pacco, N. Collaert, W. Vandervorst, and M. Caymax, Nanoscale 10, 7058 (2018).

${ }^{29}$ H. Han, T. Hantschel, A. Schulze, L. Strakos, T. Vystavel, R. Loo, B. Kunert, R. Langer, W. Vandervorst, and M. Caymax, Ultramicroscopy 210, 112922 (2020).

${ }^{30}$ K. Kuitunen, F. Tuomisto, and J. Slotte, Phys. Rev. B 76, 233202 (2007).

${ }^{31}$ V. Ranki, A. Pelli, and K. Saarinen, Phys. Rev. B 69, 115205 (2004).

${ }^{32}$ M. Ramamoorthy and S. T. Pantelides, Phys. Rev. Lett. 76, 4753 (1996). 